\title{
EL SIGNIFICADO DE CUIDADO PARA LOS ENFERMEROS QUE CUIDAN DE PERSONAS CON LESIÓN MEDULAR
}

Zuila Maria de Figueiredo Carvalho* Antonio José Núñez Hernández**, Francisco Vicente Mulet Falcó***, María Isabel Nuñez Ângulo****, Luciene de Miranda Andrade*****

*Doctora y Docente del Departamento de Enfermería de la Universidad Federal do Ceará (UFC). Hospital Universitario La Fe.

**Enfermero. Presidente de Honra do Colegio de Enfermería de Valencia - España. Miembro del Núcleo de Investigación y Extensión en Enfermería Neurológica (NUPEN/DENF/UFC.

***Enfermera de lo Servicio de lesión Medular del Hospital Universitario La Fe España.

****Doctoranda del Programa de Pos-Grado del Departamento de Enfermería de la Universidad Federal do Ceará (UFC). Enfermera del Hospital Dr. José Forta (IJF) y docente de enfermería en la Universidad Fortaleza/ Ceará/ Brasil.

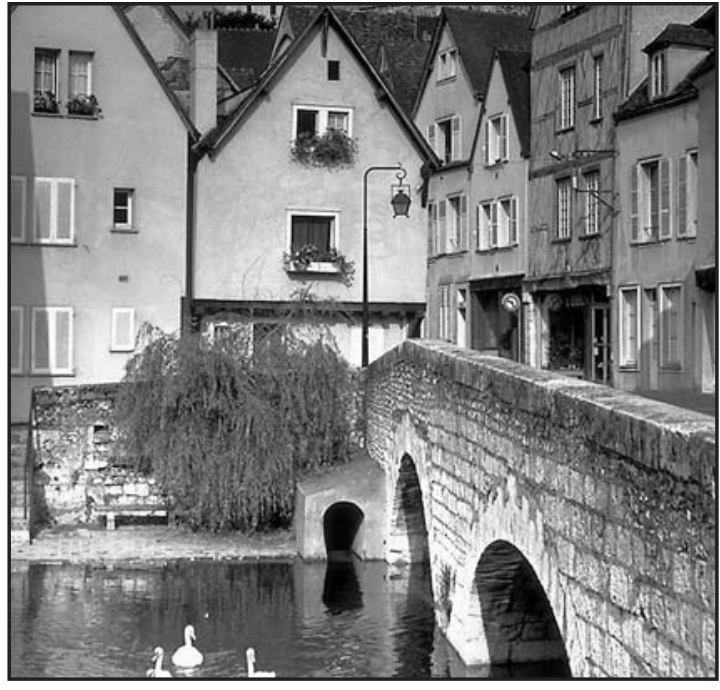

MEANING OF 'CARE' FOR NURSES WHO LOOK AFTER ILL PATIENTS WITH CORD INJURY

\section{SUMMARY}

7 he study aim was to understand what is the meaning of 'care' for nurses who look after ill patients with spinal cord injury in hospital. It is a qualitative research achieved in three hospitals, Brazilian, Spanish and Portuguese. 30 male and female nurses in charge of Medual Units were involved. 15 Brazilians, 5 Spaniards and 10 Portuguese. Data collection was gathered in 2003, 2006 and 2007, by means of structured interviews, after the signing of due terms of free Acknowledge and Consent. The main query was: What is care? Answers revealed three different thematic categories: Care: actions taken according to nurse basic human needs; Care: educational actions; and Care: attitudes and care behaviours;

The study suggests there are many coincidences in meaning for caring the so called ill patients involved (cord injury lesions), between Spanish and Portuguese nurses, towards Watson's personal meaning.

The outcome is that nurses understand that 'care' is the main point in the process or 'caring', being this perceived as nurses skillfulness to grasp the most important subjective feature of the patient.

To get a proper relationship between nurse and patient demands from the latter to experience, imagine and feel the closest to the patient. Thus, we feel transpersonal care is part of inter link to care these type of ill patients.

Key words: caring, nursing theory, understanding the meaning of 'care', cord injury. Jean Watson 


\section{O SIGNIFICADO DE CUIDADO PARA OS ENFERMEIROS QUE CUIDAM DE PESSO- AS COM LESÃO MEDULAR}

\section{RESUMO}

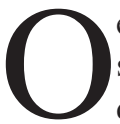

estudo teve como objetivo compreender o significado de cuidado para os enfermeiros que cuidam de pessoas com lesão medular hospitalizadas. É uma investigação qualitativa realizada em três hospitais, brasileiro, espanhol e português. Os envoltos foram 30 enfermeiros, que trabalham em Unidade Medular, sendo: 15 brasileiros, 05 espanhóis e 10 portugueses. A coleta dos dados foi realizada, os anos 2003, 2006 e 2007, por meio de entrevista estruturada, depois da assinatura do termo de Consentimento Livre e Esclarecido. Teve como questão: Que é cuidado? Os discursos desvelaram três categorias temáticas: Cuidado: ações desenvolvidas com vistas o atendimento das necessidades humanas básicas; Cuidado: ações educativas e Cuidado atitudes e comportamentos de cuidar. O estudo aponta muitas convergências nos significados de cuidados para os enfermeiros envoltos, mas, entre os espanhóis e portugueses há maior congruência nos significados de cuidados com os modos de pensar "cuidado" para Watson. Concluí-se que os enfermeiros entendem o cuidado como um importante componente no processo de cuidar. Fato este percebido como a habilidade dos enfermeiros em captar a importância subjetiva da pessoa. Relacionar-se por meio do cuidado transpessoal exige da enfermeira a capacidade de unirse, experimentar e imaginar o sentimento do paciente. Desta forma entendemos que o cuidado transpessoal faz parte da relação de cuidar de estejas enfermeiros.

Palavras Chave: cuidado, teoria de enfermagem, compreensão de significado, lesão medular, Jean Watson.

\section{RESUMEN}

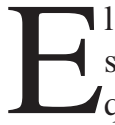
1 estudio tuvo como objetivo comprender el significado de cuidado para los enfermeros que cuidan de personas con lesión medular hospitalizadas. Es una investigación cualitativa, realizada en tres hospitales, brasileño, español y portugués. Los implicados fueron 30 enfermeros, que trabajan en Unidad Medular, siendo: 15 brasileños, 05 españoles y 10 portugueses. La colecta de los datos fue realizada, los años 2003, 2006 y 2007, por medio de entrevista estructurada, después de la firma del término de Consentimiento Libre y Esclarecido. Tuvo como cuestión: ¿Qué es cuidado? Los discursos desvelaron tres categorías temáticas: Cuidado: acciones desarrolladas con vistas la atención de las necesidades humanas básicas; Cuidado: acciones educativas y Cuidado actitudes y comportamientos de cuidar. El estudio apunta muchas convergencias en los significados de cuidados para los enfermeros implicados, pero, entre los españoles y portugueses hay mayor congruencia en los significados de cuidados con los modos de pensar "cuidado" para Watson. Concluimos que los enfermeros entienden el cuidado como un importante componente en el proceso de cuidar. Hecho este percibido como la habilidad de los enfermeros en captar la importancia subjetiva de la persona. Relacionarse por medio del cuidado transpersonal exige de la enfermera la capacidad de unirse, experimentar e imaginar el sentimiento del paciente. De esta forma entendemos que el cuidado transpersonal forma parte de la relación de cuidar de estés enfermeros.

Palabras Clave: cuidado, teoría de enfermería, comprensión del significado, lesión medular. Jean Watson.

\section{INTRODUCCIÓN}

La lesión medular es un síndrome neurológico con secuela irreversible, que lleva a las profundas modificaciones en la vida del acometido, de su familia y de la sociedad en que vive. La problemática presentada por esas personas demanda una manera de cuidar que englobe todas las dimensiones de la persona, es decir, tanto cuidados del dominio biológico, como del emocional, social, espiritual y existencial.

El cuidado es una habilidad propia de la Enfermería, desde que ella es una profesión. Compartido con el modo de pensar de Watson (1979) de lo que el cuidado es un ideal moral, un valor humano que envuelve antojo, decisión y 
compromiso para cuidar, acciones de cuidado y sus consecuencias, y que debe ayudar la persona a recoger significaciones para enfermedad, sufrimiento, dolor y existencia.

La teórica arriba citada dice que los conceptos esenciales y específicos para su modelo teórico son el cuidado transpersonal, el ego, el campo fenomenológico y la ocasión del cuidado actual. Así, el cuidado es definido como una ciencia desarrollada a partir de una filosofía humanista, la cual es el enfoque de la Enfermería (Watson, 1979).

Como es realizado el cuidado de enfermería con las personas portadoras de deficiencias ocasionadas por lesión de la médula, así como, el significado de cuidar bajo la óptica de aquellos que cuidan, ha sido motivo de preocupación y de indagaciones para nosotros, delante de estos hechos, hay mucho años estamos realizando estudios sobre esta temática.

Los últimos años se ha observado que los enfermeros tanto brasileños como extranjeros están desarrollando cada día más estudios sobre cuidado, de entre ellos destacamos: Villa y Rosse, (2001); Waldow, (2004); Collet y Roca, (2004); Leite y Faro (2005); Carvalho et al (2006a); Souza y Santos (2007); Hernández (2008) y Rocha et al (2008). Sin embargo, hay aún, una laguna en la literatura sobre cuidado en la óptica de los enfermeros, pero encontramos los de Silva (2001); Carvalho (2004) los estudios direccionados al tema cuidado con personas parapléjicas en la percepción de los enfermeros son más escasos aún.

Por todo lo expuesto esta investigación que tiene como objeto de estudio el significado de cuidado en la percepción de los enfermeros que cuidan de personas con lesión medular, se hace relevante porque es una experiencia vivida en el contexto de la diversidad cultural de cuidado, además de ser una contribución en la formación de un cuerpo de conocimiento para la práctica de la enfermería neurológica.

\section{OBJETIVO}

Comprender el significado de cuidado para los enfermeros portugueses, españoles y brasileños que cuidan de personas con lesión medular hospitalizadas y analizar a luz del modelo de cuidado transpersonal de Jean Watson.

\section{TRAYECTORIA METODOLÓGICA}

Se trata de una investigación del tipo estudios de campo, con corte longitudinal y de abordaje cualitativa. La investigación de campo "recoge la descripción y la explotación de fenómenos, en escenarios naturales" (Polit, Beck y Hungler, 2004). Son estudios realizados en locales de convivencia social, como hospitales, clínicas, unidades de tratamiento intensivo, de entre otros. Ya los estudios longitudinales acontecen en múltiples puntos de colecta de los datos durante un largo periodo de tiempo (Hulley et al. ,2007).

Fue realizada en tres hospitales de gran porte, portugués, español y brasileño que poseen Unidades de Internación para los Lesionados Medulares. Los sujetos implicados fueron 30 enfermeros de ambos sexos, que cuidan de personas con lesión medular en Unidad Medular de los referidos hospitales, siendo: 10 portugueses 5 españoles y 15 brasileños. La recogida de los datos fue en los años 2003, 2006 y 2007, por medio de entrevista estructurada, y tuvo como cuestión para los enfermeros: ¿Qué es cuidado? Fueron realizadas después de la firma del término de Consentimiento Libre y Esclarecido.

Los datos fueron organizados por la "Analice del Contenido" - Bardan (2004), esta técnica fue escogida por constituirse de un método de utilización indiscutible en las investigaciones que envuelven evaluaciones e interpretación de datos con base en elementos textuales de los discursos. Para tanto realizamos las fases básicas, pre-análisis; explotación del material y tratamiento de los resultados, inferencias e interpretación.

La ética en la investigación fue respetada en todo el recorrido de la investigación, conforme los preceptos de la Resolución n ${ }^{\circ}$ 196/96 del Consejo Nacional de Salud, (Brasil, 1996). El derecho al anonimato fue considerado, tanto los enfermeros serán identificados por abreviaturas, Enf.B para los brasileños, Enf.E, los españoles y Enf.P, los portugueses.

De los discursos de los enfermeros fueron desveladas tres categorías, a saber, Cuidado: acciones desarrolladas con vistas la atención de las necesidades humanas básicas; Cuidado: acciones educativas y Cuidado: actitudes y comportamientos de cuidar. El análisis fue a la luz de la Teoría del 
Cuidado Transpersonal de Jean Watson, 1985 1999.

\section{Los hallazgos:}

\section{Cuidado: acciones desarrolladas con vistas la} atención de las necesidades humanas básicas

A lo que expresen el significado de cuidado en el contexto estudiado, las enfermeras se reportan a éste como siendo acciones direccionados para la satisfacción de las necesidades básicas. A despecho de la abrangencia que esta expresión traduce, es notorio el énfasis que es dada a la atención de las necesidades del dominio biológico, a lo que digan que cuidado es:

"Dar baño, realizar cateterismo vesical, alimentar, administrar la terapéutica medicamentosa, cambiar de decúbito, cuidar de la piel, hacer curativo, de entre otros". (Enf.B).

Se percibe que las enfermeras portuguesas y españolas, no niegan las otras dimensiones del cuidado, resaltando las necesidades emocionales, el dialogo y la atención a la familia, como ilustran las declaraciones.

"Cuidado es satisfacer las necesidades básicas de los enfermas. Es la prestación de cuidados, desde la higiene, es dar apoyo emocional, es conversar con ellos. Por lo tanto, no es sólo en términos de cuidar de los aspectos físicos del enfermo". (Enf.P)

"Cuidado es tratar de sus necesidades físicas, también las psicológicas, tratar también sus familiares y todas las cosas". (Enf.E)

Estas enfermeras se posicionaron de esta forma: tienden a privilegiar el lado de la intersubjetividade y de la trascendencia, conforme sugerido por Watson (2002) aunque no consigan entrever tales aspectos desvinculados de la realización de procedimientos técnicos.

Carvalho, (2002) apunta que este momento de trascendencia, que acontece a través de la interacción, de la actitud, del diálogo y de la preocupación con los familiares, se revela en una serie de significados para los envueltos en el cuidado.

En la teoría del Cuidado Transpersonal, Watson (1988) muestra que el foco nuclear de la Enfermería está centrado en el hecho de que el cuidado "se deriva de una perspectiva humanística, combinada con una base de conocimientos cientí- ficos". Para la teórica, cuidado humano es un ideal moral, que trasciende al acto de una enfermera aisladamente y produce actos efectivos de la Enfermería como profesión para la realización humana.

Incluye, por lo tanto, atención individual y preocupación, consideración y cariño. Independiente del punto de vista cultural, el cuidado puede ser considerado como "atención”, "preocupación para", "responsabilidad por”, "observar con atención”, "con afecto, amor, y simpatía”. Es decir, el término implica en la idea de hacer, de acción.

\section{Cuidado: acciones educativas}

En los discursos de las enfermeras, se percibe que los cuidados tienen, también, el significado de acciones diseccionadas para las actividades de enseñanza/aprendizaje, esto se queda evidente cuando ellas se posicionan:

"La enseñanza es todo aquello que pueda ayudar en el futuro, la persona que va a necesitar de ayuda en esa altura, todo aquello que pueda colaborar para su mejoría, todo eso forma parte del cuidado, puede ser así directo o indirecto" (Enf.P)

"Es enseñanza auto cuidados a los enfermos y sus familiares; enseñanza de cambios posturales, alimentación" (Enf.E)

"Es enseñanza del cateterismo de alivio intermitente (CAT), actividades de cambios de decúbito, importancia de una dieta equilibrada y de la ingestión de líquidos, y, aún, sobre cuidados para prevenir escaras además de las relativas a la orientación sexual" (Enf.B).

La promoción de la enseñanza-aprendizaje es un factor de cuidado propuesto en la teoría del cuidado Transpersonal (Watson, 1988 y 1999). La teórica alerta a las enfermeras, en el sentido de mostrar que este, es un factor propiciador a las personas el máximo de control sobre su propia salud, porque él ofrece informaciones y alternativas, además de favorecer mayor cambios entre enfermera y paciente.

Es evidente el interés de las enfermeras en lo que se refiere a la enseñanza. Se percibe, sin embargo, que tal enseñanza insiste en privilegiar las cuestiones eminentemente físicas, contrariando de esta forma lo que es sugerido por Watson (1988). 


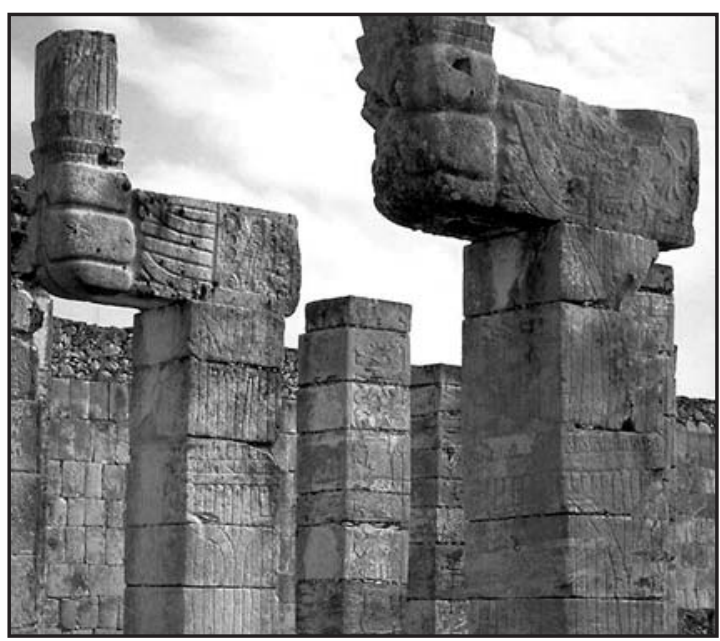

Acerca de este asunto, Carvalho et al (2006b) en una investigación "la enseñanza del sondaje limpio intermitente - una estrategia de cuidado", concluyen que enseñar a la persona enferma es un desafío, pero hacerlo en situaciones especificas tales como las personas debilitadas emocionalmente como es el caso de los lesionados medulares es un desafío aún, mayor.

Llama la atención la autora arriba citada en un estudio sobre la experiencia de promover enseñanza y aprendizaje de cuidados, también a los familiares de las personas con discapacidad (Carvalho et al 2006c).

\section{Cuidado: actitudes y comportamientos de cui- dar}

Ya existe consenso, entre las enfermeras, al identificar la meta de la enfermería en el proceso de cuidado es ayudar las personas a que alcancen un alto grado de armonía dentro de sí, de forma a promover el autoconocimiento y la propia cura, o para promover insight del significado de los acontecimientos de la vida. En este sentido dice Queiroz (2004) que las situaciones de cuidados se transforman en ayudar a vivir.

Los discursos de los sujetos implicados muestran que cuidado es también, actitudes y comportamientos de cuidar, y que en estos se integran sentimientos diversificados, noten lo que fue hablado:

"Es hacer las cosas sin reclamar, es dar atención, cariño, es amor, es también, cualificación, preocupación y atención. No es solamente dar una inyección y aliviar aquel dolor. A las veces, sólo una persona hablará, la otra ya se siente más confortable, puede hasta aliviar un dolor". (Enf.P)

"Es preocupación, cariño, dedicación, diálogo, y celo. Es en las horas difíciles, conversar con el enfermo, por encima de todo, gustar de lo que hace. Es dar atención, cariño, es amor, es también, cualificación, preocupación y atención" (Enf.E)

"Es conversar, esclarecer dudas, ayudar, incentivar, enseñar, dar amor, envolverse y demostrar interés y realizar los procedimientos técnicos con amor e interés”. (Enf. B).

Para las enfermeras de este estudio, es destacable como cuidado las actitudes, la preocupación, cariño y amor, son vistas como acciones de cuidado. De cierta forma, esas enfermeras incorporan en sus modos de cuidar las características de la empatía y de la relación de ayuda.

Esas actitudes y comportamientos de cuidar evidenciadas ayudan a la enfermera a comprender el significado que la persona da a su vida, o aún, puede ayudar a la persona a encontrar sentido en los acontecimientos difíciles, contribuyendo también a que la persona encuentre la fuerza o el coraje para enfrentar la vida a pesar de la deficiencia.

A ese respecto, Queiroz (2004) es enfático al decir que la relación de ayuda en el contexto del cuidado es un elemento decisivo para la incorporación de intervenciones interdependientes y autónomas bien fundamentadas desde el punto de vista científico, técnico y ético.

De ese modo, entendemos que la empatía y la relación de ayuda deben fundamentarse para que los cuidados de enfermería sean realmente eficaces.

Watson (2006) recuerda que cuidar es una habilidad propia de la enfermería, y que la enfermera siempre estuvo preocupada con la posición del cuidar humano, en lo que concierne a las situaciones de salud/enfermedad de las personas. Como, tan bien, nos dice Colière, (2001) cuidar es tener en cuenta las dos caras de los cuidados, lo que ofrece cuidado y lo que recibe este cuidado.

\section{DISCUSIÓN}

El estudio apunta algunas convergencias en los significados de cuidados para los enfermeros implicados, pero, entre los enfermeros españoles y portugueses hay mayor congruencia en los signifi- 
cados de cuidados con los modos de pensar "cuidado" para Watson.

Para Watson, el cuidado es el atributo más valioso que la Enfermería tiene para ofrecer a la humanidad, pues incorpora valores al cuidado humano, apuntando determinadas condiciones para que este sea eficiente. Tales condiciones están descritas en las premisas de la ciencia del cuidado.

Es evidente el interés de las enfermeras en lo que se refiere a la enseñanza-aprendizaje. Pero, tal enseñanza insiste en privilegiar las cuestiones eminentemente físicas, contrariando lo que es sugerido por Watson. Sin embargo, algunas enfermeras diseccionan sus cuidados para actitudes de empatía y de la relación de ayuda.

Esos hallazgos denotan que las enfermeras que cuidan de los parapléjicos aún no incorporaron de todo, en su práctica, el nuevo paradigma sobre cuidado humano, mostrando que tienen delante de sí el desafío de pensar el cuidado de forma diferenciada.

\section{CONSIDERACIONES FINALES}

Los enfermeros implicados en el estudio entienden el cuidado como un importante componente en el proceso de cuidar. Hecho este percibido como la habilidad de los enfermeros en captar la importancia subjetiva de la persona.

Relacionarse por medio del cuidado transpersonal exige de la enfermera la capacidad de unirse, experimentar e imaginar el sentimiento del paciente.

El estudio muestra que los enfermeros no niegan las otras dimensiones del significado de cuidado, resaltando las sociales, emocionales, espirituales y existenciales. De esta forma entendemos que el cuidado Transpersonal forma parte de la relación de cuidar de estos enfermeros.

Una consideración que podemos hacer es que el conocimiento científico y el conocimiento humanístico son esenciales para la ciencia y para el arte de la Enfermería. La dimensión fenomenológica de experiencias humanas en la salud y en la enfermedad proveerá los enfermeros con datos necesarios para entender la condición humana y los factores curativos elaborados por Jean Watson.

La investigación muestra que hoy se hace necesario que las enfermeras compartan el compromiso con el avance de la ciencia de enfermería a través de investigación y de la utilización de sus hallazgos en la práctica, así, el conocimiento obtenido es transformado en practica clínica, culminando en una Enfermería vinculada a la investigación.

\section{AGRADECIMIENTOS Y AYUDAS:}

Estudio realizado en el ámbito del Proyecto de Investigación "Cuidados con las personas con problemas neurológicos" del Programa de Postgrado en Enfermería de la Universidad Federal de Ceará. Presentado en el VI jornadas internacionales de cultura de los cuidados Familia, Cultura y Cuidados y IX Reunión de Investigación Cualitativa da Universidad de Alicante, 12 y 13 de Junio de 2008. Tuvo apoyo financiero del Colegio de Enfermería de Valencia - España

\section{BIBLIOGRAFÍA}

- Bardan L. (1979 Análise de conteúdo. Lisboa: Edições 70 Ltda.

- Brasil, MS (1996) Conselho Nacional de Saúde. Resolução 196/96. Brasília: CSN.

- Carvalho ZM de F. (2002) Cuidado de enfermagem com pessoas paraplégicas hospitalizadas: estudo à luz da teoria de Jean Watson. Tese (Doutorado em Enfermagem) - Universidade Federal do Ceará, Programa de Pós-Graduação em Enfermagem, Fortaleza.

- Carvalho ZM de F. (2004) O cuidado de enfermagem dirigido a pessoas com lesão vértebro medular. Interacções, vol. 6 , n. 1, p.175-83, abr.

- Carvalho ZM de F. y et. al (2006 $)$ O Cuidado Solidario: una estrategia para el cuidado de las personas con lesión medular. Revista Educare 21, Espanha, v. 28, n. Junho, p. 1-10.

- Carvalho ZM de F. et al. (2006b) La enseñanza del sondaje limpio intermitente - una estrategia de cuidado. Investigación \& Cuidados, Alicante, v. 4, n. $2^{\circ}$, p. 37-42.

- Carvalho ZM de F. y et. al. (2006c) Pacientes com lesão raquimedular: experiência de ensino-aprendizagem do cuidado para suas famílias. Esc. Ana Nery, vol.10, n. 2, ago, p 316-322.

- Collière M-F. (2001) soigner ... le premier art de la vie. $2^{\mathrm{a}}$ edição. Paris, Masson.

- Collet N. y Rocha SMM. (2004) Criança hospitalizada: mãe e enfermagem compartilhando o cuidado. Rev. Latino-Am. Enfermagem, Mar./Apr., vol.12, no.2, p.191-197.

- De Figueiredo Carvalho ZM y Coelho Damasceno MM. (2003) Aplicación de la teoría del cuidado transpersonal en pacientes parapléjicos hospitalizados: relato de experiencia. Cienc. Enferm., dic. vol.9, no.2, p.77-94.

- Hernández MHF y Juan LC. (2008) El aprendizaje de cuidados familiares a pacientes com Alzheimer. Revisión Bibliográfica.Rev. Cultura de los Cuidados, 1er. Semestre. Ano XII - No 23: 57- 69.

- Hulley SB y et al.(2007) Designing Clinical Research. 3rd Edition. Lippincott Williams \& Wilkins. USA.

- Leite VB y FARO ACM.. (2005) O cuidar do enfermeiro especialista em reabilitação físico-motora. Rev. Esc. Enf. USP, vol.39, n. 1, p. 92-6. 
- Polit DF , Bech CT y Hungler BP. (2004) Fundamentos de pesquisa em Enfermagem. Tradução Regina Machado Garcez. Porto Alegre: Artes Médicas.

- Queiróz AA .(2004) Empatia e Respeito. 2 ${ }^{\mathrm{a}}$ Ed. Coimbra: Editora Ariane.

- Rocha PK, Prado ML, Wal ML, Carraro TE . (2008) Cuidado e tecnologia: aproximações através do Modelo de cuidado. Rev Bras Enferm, Brasília, jan-fev; 61(1): 113-6.

- Silva LF da, Damasceno MMC, Carvalho CML, Souza PDS de. (2001) Cuidado de enfermagem: o sentido para enfermeiros e pacientes. Rev. bras. enferm ;54(4):578-588, out.-dez.

- Sousa PML y Santos IMSC. (2007) Cuidando da pessoa com ferida cirúrgica: estudo de caso. Rev. Referência II ${ }^{\mathrm{a}}$ Série, n. 4. - Vila VSC y Rossi LA. (2002) Significado cultural del cuidado humanizado en la unidad de terapia intensiva: "mucho se habla y poco se vive". Rev. Latino-Am. Enfermagem, Mar./Apr. vol.10, no.2, p.137-144.
- Waldow VR. (2004) Cuidar expressão humanizadora da enfermagem. Rio de Janeiro: Vozes.

- Watson J. (1979) Nursing: the philosophy and science of caring. Publisher: University Press of Colorado; Reprint edition.

- Watson J. (1985) Nursing: human science and human care, a theory of nursing, New York: National League for Nursing.

- Watson J. (1988) Nursing: human science and human care, a theory of nursing, New York: National League for Nursing.

- Watson J. (1999) Nursing: human science and human care, a theory of nursing. Denver: Publisher Jones \& Bartlett Publishers.

- Watson J. (2006) Carative factors - Caritas processes guide to professional nursing. Danish Clinical Nursing Journal. 20(3), 21-271.

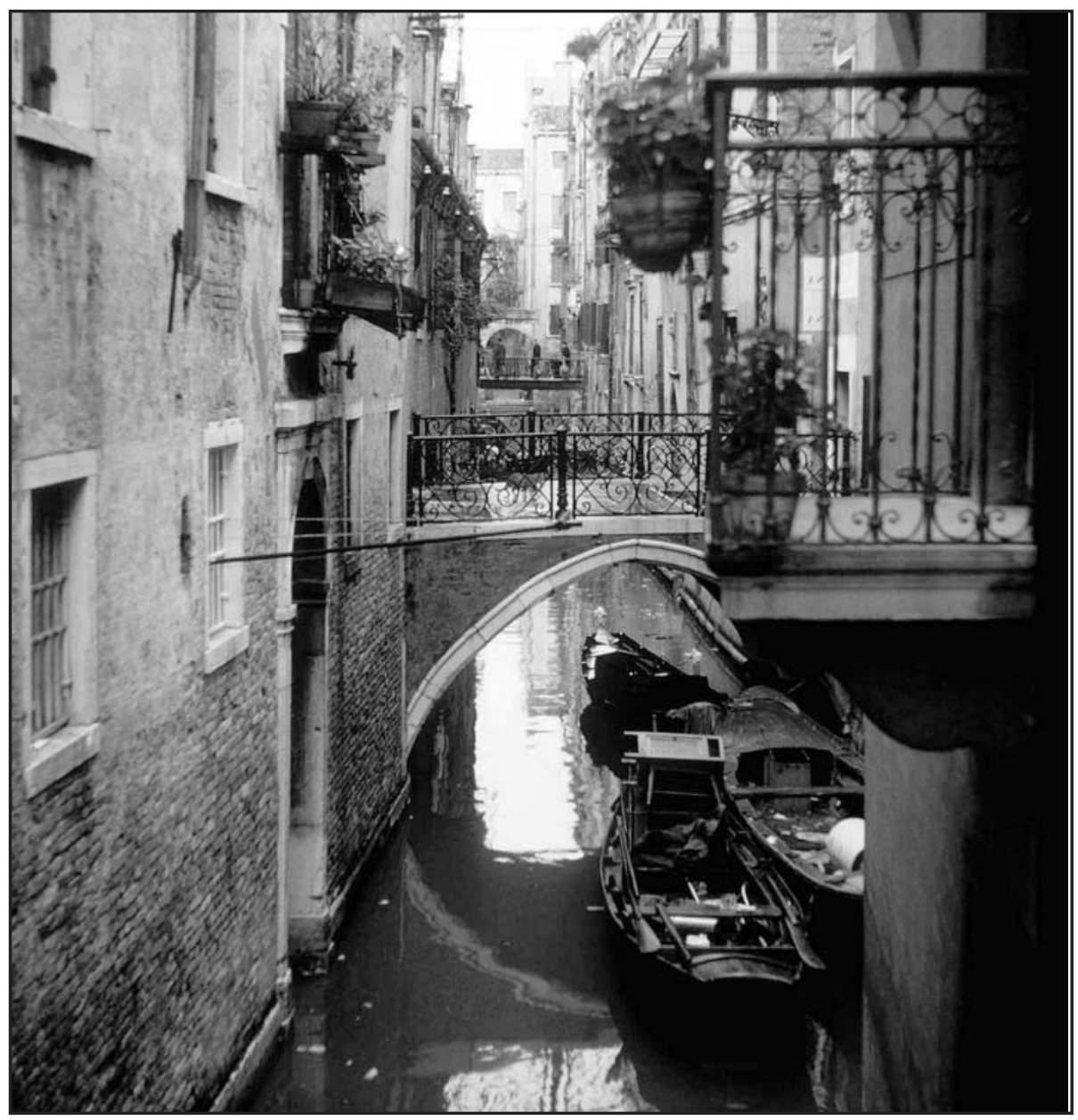

